\title{
Attractive Forces between Electrons in (2+1)-Dimensional QED
}

\author{
H. O. Girotti, ${ }^{(a)}$ M. Gomes, J. L. deLyra, R. S. Mendes, and J. R. S. Nascimento \\ Instituto de Física, Universidade de São Paulo, Caixa Postal 20516, 01498 São Paulo, São Paulo, Brazil
}

\begin{abstract}
A. J. da Silva ${ }^{(b)}$
Center for Theoretical Physics, Massachusetts Institute of Technology, Cambridge, Massachusetts 02139

(Received 29 May 1992)
\end{abstract}

\begin{abstract}
Vacuum polarization effects are nonperturbatively incorporated into the photon propagator to eliminate the severe infrared problems characteristic of $(2+1)$-dimensional QED $\left(\mathrm{QED}_{3}\right)$. The theory is thus rephrased in terms of a massive vector boson whose mass is $e^{2} / 8 \pi$. Subsequently, it is shown that electron-electron bound states are possible in $\mathrm{QED}_{3}$.
\end{abstract}

PACS numbers: 11.15.Tk, 11.10.St, 12.20.Ds

Recently [1], the effective nonrelativistic potential

$$
\begin{aligned}
V(\overrightarrow{\mathrm{r}})= & \frac{e}{2 \pi}\left(1-\frac{\theta}{m}\right) K_{0}(|\theta| r) \\
& -\frac{e}{\pi \theta} \frac{1}{m r^{2}}\left[1-|\theta| r K_{1}(|\theta| r)\right]_{L},
\end{aligned}
$$

describing the fermion-fermion interaction in the Maxwell-Chern-Simons (MCS) theory [2], was derived in the lowest perturbative order [the notation in Eq. (1) is explained in Ref. [3]] [4]. The aim of Ref. [1] was to determine whether the potential (1) could bind a pair of identical fermions. For positive values of $\theta$, a numerical solution of the Schrödinger equation confirmed the existence of a bound state for $e^{2} / \pi \theta=500, m / \theta=10^{5}$, and $l=1$. Further numerical analysis indicated that all identical-fermion bound states are located in the region $e^{2} / \pi \theta>1$.

Thus, we were naturally led to study the limit $\theta \rightarrow 0$ where the MCS theory degenerates into $(2+1)$ dimensional QED $\left(\mathrm{QED}_{3}\right)$. Power counting indicates that $\mathrm{QED}_{3}$ is plagued with infrared singularities whose degree of divergence grows with the order of perturbation. Moreover, when $\mathrm{QED}_{3}$ is treated nonperturbatively by means of the Bloch-Nordsieck (BN) approximation [5-7], one finds for the two-point fermionic Green's function of momentum $p$ the expression

$$
G\left(p ; m_{R}\right)=-\frac{i}{|Q|} \int_{0}^{\infty} d v \exp \left\{\epsilon(Q)_{v}-\frac{e^{2}}{4 \pi|Q|} v\left[C+\ln \left(\frac{M}{|Q|} v\right)\right]\right\},
$$

where $Q \equiv u \cdot p-m, u$ is a timelike vector $\left(u^{2}=1\right)$ replacing the gamma matrices in the $\mathrm{BN}$ scheme, $\epsilon(Q)$ is the sign function, $M$ is a subtraction point, and $C$ is the Euler constant. Clearly, $G$ is well defined for generic values of $Q$ but develops an essential singularity at $Q=0$. The fact that $G$ does not behave as a simple pole at $Q=0$ signals the presence of infrared singularities.

As is known [5], the BN approximation eliminates all vacuum polarization diagrams [8]. In this work we start by demonstrating that when the vacuum polarization effects are nonperturbatively incorporated into the phonon propagator, the infrared structure of $\mathrm{QED}_{3}$ changes drastically and all inconsistencies disappear. Essentially, the theory is reformulated in terms of a massive vector boson whose mass is $|\theta|=e^{2} / 8 \pi$, the dynamically induced Chern-Simons term [2,9] being at the root of this mechanism. It turns out then that the effective electronelectron low-energy potential arising from $\mathrm{QED}_{3}$ can be read off directly from (1) after the replacement $\theta$ $\rightarrow-e^{2} / 8 \pi$, namely,

$$
\begin{aligned}
e V^{\mathrm{QED}_{3}}(\overrightarrow{\mathrm{r}})= & \frac{e^{2}}{2 \pi}\left(1+\frac{e^{2}}{8 \pi m}\right) K_{0}\left(\frac{e^{2}}{8 \pi} r\right) \\
& +\frac{8}{m r^{2}}\left[1-\frac{e^{2}}{8 \pi} r K_{1}\left(\frac{e^{2}}{8 \pi} r\right)\right] L .
\end{aligned}
$$

The terms proportional to $K_{0}$ in (3) are now both repulsive. The term $8 L / m r^{2}$ becomes attractive (repulsive) for negative (positive) eigenvalues of $L$, while the term proportional to $K_{1}$ acts in the opposite way. We conclude the paper by showing that electron-electron bound states are also possible in $\mathrm{QED}_{3}$.

Our starting point is the $\mathrm{QED}_{3}$ Lagrangian density [10]

$$
\begin{aligned}
\mathcal{L}= & -\frac{1}{4} F_{\mu \nu} F^{\mu \nu}-\frac{1}{2 \lambda}\left(\partial_{\mu} A^{\mu}\right)\left(\partial_{v} A^{\nu}\right)+\frac{i}{2} \bar{\psi} \gamma^{\mu} \partial_{\mu} \psi \\
& -\frac{i}{2}\left(\partial_{\mu} \bar{\psi}\right) \gamma^{\mu} \psi+e \bar{\psi} \gamma^{\mu} A_{\mu} \psi-\bar{m} \bar{\psi} \psi,
\end{aligned}
$$

describing the coupling of charged fermions $(\bar{\psi}, \psi)$ of mass $m=|\bar{m}|$ and charge $e$ to the gauge field potential $A^{\mu}$. In principle, $\bar{m}$ can be either positive or negative, but we shall analyze here the case $\bar{m}>0$. Neither parity nor time reversal are, separately, symmetries of the model.

We concentrate on the lowest-order graph contributing to the vacuum polarization tensor $\Pi^{\rho \sigma}(q)$. Since we are interested in the quantum corrections to a nonrelativistic potential, we shall retain only those terms of zero and first order in $q$. Gauge invariance alone ensures that $\Pi^{\rho \sigma}(0)=0$. As for the first-order contribution, which gives origin to the induced Chern-Simons term [2,9], one 
finds

$$
\Pi_{\rho \sigma}^{(1)}(q)=-i\left(e^{2} / 8 \pi\right) \epsilon_{\rho \sigma \mu} q^{\mu} .
$$

We emphasize that $\Pi_{\rho \sigma}^{(1)}(q)$ is ultraviolet finite, and that, therefore, no regularization is needed for its computation. At this point a word of caution is necessary. If one adopts the point of view of keeping ultraviolet divergences under control by regularizing the entire theory, the result quoted in this last equation is only true if a parity-timereversal-invariant regularization is used.

Because of the severe infrared singularities, the standard perturbative series fails to exist in $\mathrm{QED}_{3}$. On the other hand, one knows that when a Chern-Simons term is added to the free part of the $\mathrm{QED}_{3}$ Lagrangian (the MCS theory), a topological mass for the vector field is generated, freeing the theory from the infrared divergences. In view of this, we modify the photon propagator by resumming the geometric series resulting from the iteration of $\Pi_{\rho \sigma}^{(1)}$ (see Fig. 1). As far as the nonrelativistic approximation is concerned, this is equivalent to the introduction into the free Lagrangian of the Chern-Simons term

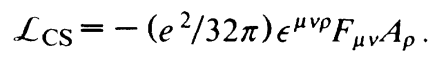

Hence, the induced Chern-Simons coefficient is, as previously stated,

$$
\theta_{\text {in }}=-e^{2} / 8 \pi \text {. }
$$

From (1) and (7) it then follows that the $\mathrm{QED}_{3}$ effective electron-electron low-energy potential is, in fact, that given by (3).

Before investigating whether the potential (3) can sustain electron-electron bound states, we want to show that the above proposed solution for $\mathrm{QED}_{3}$ is consistent, in the sense that all remaining contributions to $V^{\mathrm{QED}_{3}}$ are, up to some power of $\ln \left(e^{2} / m\right)$, of order $e^{2} / m$ or higher with respect to (3) and, therefore, vanish as $e^{2} / m \rightarrow 0$. To see how this comes about, we compute the vertex correction $\Lambda^{\mu}$ to $V^{\mathrm{QED}_{3}}$ arising from the diagram in which only one massive vector particle is exchanged (see Fig. 2) [11]. After the replacement $\theta \rightarrow \theta_{\text {in }}$, the massive vector field propagator can be read off directly from Eq. (4) of Ref. [1],

$D_{\mu v}(k)=\frac{-i}{k^{2}-\theta_{\mathrm{in}}^{2}}\left(P_{\mu v}-i \theta_{\mathrm{in}} \epsilon_{\mu v \rho} \frac{k^{\rho}}{k^{2}}\right)-i \lambda \frac{k_{\mu} k_{v}}{k^{4}} f\left(k^{2}\right)$,

where $P_{\mu \nu} \equiv g_{\mu \nu}-k_{\mu} k_{v} / k^{2}$ and an arbitrary function $f\left(k^{2}\right)$ has been incorporated into the longitudinal part. Accordingly, $\Lambda^{\mu}$ can be split as follows:

$$
\Lambda^{\mu}=\Lambda_{g}^{\mu}+\Lambda_{\epsilon}^{\mu}+\Lambda_{L}^{\mu}
$$

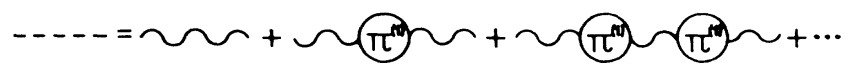

FIG. 1. Wavy lines represent free photons, while dashed lines refer to massive vector particles. where the subscripts $g, \epsilon$, and $L$ make reference to those pieces of $D_{\mu \nu}$ proportional to $g_{\mu v}, \epsilon_{\mu v \rho} k^{\rho}$, and $k_{\mu} k_{v}$, respectively. The computation of $\Lambda_{L}^{\mu}$ is straightforward and yields

$$
\Lambda_{L}^{\mu}=i \lambda \bar{v}^{(+)}\left(\mathbf{p}_{1}^{\prime}\right) \gamma^{\mu} v^{(-)}\left(\mathbf{p}_{1}\right) \int \frac{d^{3} k}{2 \pi^{3}} \frac{f\left(k^{2}\right)}{k^{2}},
$$

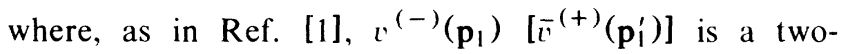
component spinor describing a free electron of twomomentum $\mathrm{p}_{1}\left(\mathrm{p}_{1}^{\prime}\right)$ in the initial (final) state. Since $\Lambda_{L}^{\mu}$ does not depend upon the momentum transfer $q \equiv p_{1}^{\prime}-p_{1}$, it can be absorbed into the wave-function renormalization constant. When computing $\Lambda_{g}^{\mu}$ and $\Lambda_{\epsilon}^{\mu}$ only zero- and first-order terms in $q / m$ will be retained, since our interest is restricted to the nonrelativistic regime. Furthermore, all loop momentum integrals are ultraviolet finite and there is, then, no need for regularization. After absorbing the zero-order terms into the wave-function renormalization constant, one arrives at

$\Lambda_{g}^{\mu(1)}(q)=\frac{N_{g}}{16 \pi}\left(\frac{e^{2}}{m}\right) \epsilon^{\mu v \rho}\left(\frac{q_{v}}{m}\right) \bar{c}^{(+)}\left(\mathbf{p}_{1}^{\prime}\right) \gamma_{\rho} v^{(-)}\left(\mathbf{p}_{1}\right)$,

$\Lambda_{\epsilon}^{\mu(1)}(q)=-\frac{N_{\epsilon}}{16 \pi}\left(\frac{e^{2}}{m}\right) \epsilon^{\mu v \rho}\left(\frac{q_{v}}{m}\right) \bar{v}^{(+)}\left(\mathbf{p}_{1}^{\prime}\right) \gamma_{\rho} v^{(-)}\left(\mathbf{p}_{1}\right)$

where

$N_{g}=-3+3 \frac{e^{2}}{8 \pi m}+2\left(1-\frac{3 e^{4}}{(16 \pi m)^{2}}\right) \ln \left(1+\frac{16 \pi m}{e^{2}}\right)$,

and

$$
N_{\epsilon}=-\frac{e^{2}}{\pi m} \ln \left(1+\frac{16 \pi m}{e^{2}}\right) \text {. }
$$

From Eqs. (11)-(14) it follows that $\Lambda^{\mu(1)}(q)$ behaves as $\left(e^{2} / m\right) \ln \left(e^{2} / m\right)$ when $e^{2} / m \rightarrow 0 \quad\left(\theta_{\text {in }} / m \rightarrow 0\right)$, while power counting indicates that the full vertex insertion $\Lambda^{\mu}(q)$ (Fig. 2) diverges logarithmically at the abovementioned infrared limit. The fact that $\Lambda^{\mu(1)}(q)$ exhibits an improved infrared behavior is not a peculiarity of the particular insertion under analysis but applies to any vertex part involving an arbitrary number of exchanged massive vector particles. Indeed, the leading infrared diver-

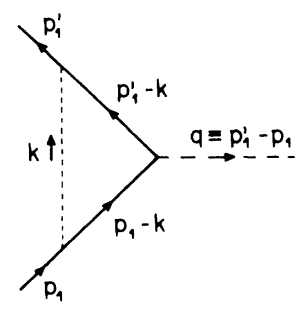

FIG. 2. The vertex insertion $\Lambda^{\mu}$. 
gence of any of these parts only shows up in those terms containing even powers of the momentum transfer $q$, as can be seen by setting to zero the loop momenta in the numerators of the corresponding Feynman integrals. Thus, the terms linear in $q$ exhibit a milder infrared behavior.

By using the technique described in [1] one finds the correction $\Delta V^{\mathrm{QED}_{3}}$ to the potential (3) arising from the diagrams in Fig. 3,

$$
e \Delta V^{\mathrm{QED}_{3}}=\left(\frac{e^{2}}{8 \pi}\right)\left(\frac{e^{2}}{m}\right)\left(N_{g}-N_{\epsilon}\right)\left[\frac{e^{2}}{16 \pi^{2} m}\left(1+\frac{e^{2}}{8 \pi m}\right) K_{0}\left(\frac{e^{2}}{8 \pi} r\right)-\frac{\delta^{2}(\mathbf{r})}{m^{2}}+\frac{e^{2}}{8 \pi^{2} m^{2}} K_{1}\left(\frac{e^{2}}{8 \pi} r\right) \frac{L}{r}\right] .
$$

From (13)-(15) it follows that $e \Delta V^{\mathrm{QED}_{3}}$ also behaves as $\left(e^{2 / m}\right) \ln \left(e^{2} / m\right)$ when $e^{2} / m \rightarrow 0$, and turns out to be negligible if $e^{2} / m \ll 1$. This establishes the region of validity of our results.

We turn next to the investigation of the existence of bound states of two identical fermions of mass $m$ interacting through the nonrelativistic potential $V^{\mathrm{QED}_{3}}$ given by (3). The corresponding radial Schrödinger equation is found to read

$$
\mathscr{H}_{l} R_{n l}(y)=\epsilon_{n l} R_{n l}(y),
$$

where

$$
\begin{aligned}
& \mathscr{H}_{l} R_{n l}(y)=-\left(\frac{\partial^{2} R_{n l}}{\partial y^{2}}+\frac{1}{y} \frac{\partial R_{n l}}{\partial y}\right)+U_{l}^{\mathrm{eff}} R_{n l}, \\
& U_{l}^{\mathrm{eff}}(y)=\frac{l^{2}}{y^{2}}+\frac{\alpha_{\text {in }}}{2}\left(1+\beta_{\text {in }}\right) K_{0}(y)+\frac{\alpha_{\text {in }} l}{y^{2}}\left[1-y K_{1}(y)\right],
\end{aligned}
$$

$y=e^{2} r / 8 \pi, \alpha_{\text {in }}=e^{2} / \pi\left|\theta_{\text {in }}\right|=8, \beta_{\text {in }}=m /\left|\theta_{\text {in }}\right|=8 \pi m / e^{2}, \epsilon_{n l}$ $=64 \pi^{2} m E_{n i} / e^{4}$, and $E_{n l}$ is the energy eigenvalue. A straightforward analysis of (18) reveals that electronelectron bound states are only possible for $l=-1,-3$, -5 , and -7 . Had we chosen the negative sign for $\bar{m}$ we would have obtained $\theta_{\text {in }}=+e^{2} / 8 \pi$ and bound states, with identical energy eigenvalues, for $l=+1,+3,+5$, and +7 .

The existence of bound states for the potential given in (18) was examined numerically by means of a stochastic variational algorithm. In this way we were able to quickly identify the state of minimum energy $(n=0)$ for a given $l$ and a variety of values of the parameter $\beta_{\text {in }}$.

In order to be able to represent the wave function $R_{0 l}(y)$ and the expectation value of the energy $\epsilon_{0 l}$ numerically, we must choose a discrete collection of points along the radial direction. Hence, we calculated

$$
\epsilon_{0 l}=\frac{\sum_{i}^{i_{\max }} \Delta y y_{i}\left[\left(\Delta R_{0 l} / \Delta y\right)_{i}^{2}+U_{l, i}^{\mathrm{eff}} R_{0 l, i}^{2}\right]}{\sum_{i}^{i_{\max }} \Delta y y_{i} R_{0 l, i}^{2}},
$$

where the values of $\Delta y$ and $i_{\max }$ were chosen to ensure a big enough range of integration, and a small enough integration interval. We then varied the wave function randomly, accepting any changes that decreased the energy [12]. We found that, starting from an arbitrary initial guess for $R_{0 l}$ it relaxed very fast towards a stable func- tional configuration. The expectation value of the energy soon became negative, confirming the existence of a bound state. One of these configurations, together with the corresponding potential, is displayed in Fig. 4.

We also measured the expectation value of the radius in the state thus obtained, which gives an idea of its size. The stability of the results was tested against variations of the range of integration interval.

In Table I we present our numerical results, calculated assuming $m$ to be the electron mass. The energy eigenvalues for $l=-5$ and -7 are very close to those corresponding to $l=-3$ and -1 , respectively, and for this reason they have not been included in Table $I$. The average radius of the bound state is given in $\AA$, and the binding energy in eV. The corresponding approximate dissociation temperature in $\mathrm{K}$ is also given. The errors quoted were evaluated from the small variations observed in the results when the range and interval of integration were varied.

It is interesting to note that there are ranges of the parameters $l$ and $\beta_{\text {in }}$ where the results are numerically consistent with the observed transition temperatures of high-

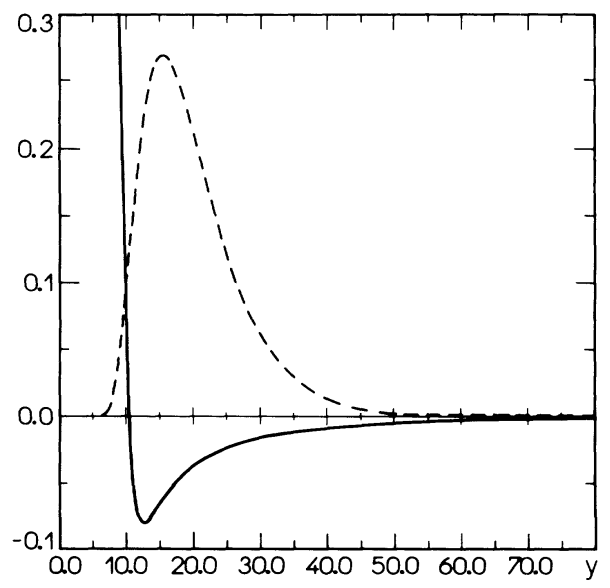

FIG. 4. The bound state wave function (dashed line) and the potential $U_{l}^{\text {eff }}(y)$ (solid line) for $l=-3$ and $\beta_{\text {in }}=3000$. The vertical scale refers only to the potential. The normalization of the wave function is arbitrary. 
TABLE I. The size and energy of the bound states.

\begin{tabular}{rrrcc}
\hline \hline$l$ & $\beta_{\text {in }}$ & Size $(\AA)$ & Energy $\left(10^{-3} \mathrm{eV}\right)$ & Temperature $(\mathrm{K})$ \\
\hline-1 & 1000 & $88 \pm 2$ & $-4.8 \pm 0.1$ & $55 \pm 1$ \\
& 3000 & $300 \pm 5$ & $-0.42 \pm 0.01$ & $4.8 \pm 0.1$ \\
-3 & 1000 & $64 \pm 1$ & $-19.6 \pm 0.4$ & $228 \pm 4$ \\
& 3000 & $218 \pm 4$ & $-1.7 \pm 0.04$ & $19.7 \pm 0.4$ \\
\hline \hline
\end{tabular}

$T_{c}$ superconductors. We do not think that this coincidence is merely accidental, although we are far from claiming that $\mathrm{QED}_{3}$ appropriately describes all features of high- $T_{c}$ superconductivity. Notice that if the initial state of the system only contains low-energy electrons, energy conservation forbids the production of electronpositron pairs and, hence, only electron-electron bound states can be formed. No electron-positron bound state can show up and, as a consequence, only the electronelectron bound state may serve to characterize the many-body ground state. Of course, it remains to be shown that the theory exhibits a phase transition and that the many-body ground state is a condensate of electronelectron pairs. However, if this turns out to be the case we find it reasonable to think that some of the dissociation temperatures given in this paper should at least be a rough approximation for the true transition temperatures.

We acknowledge Professor R. Jackiw for reading the manuscript and for many valuable criticisms. One of us (A.J.S.) thanks the Center for Theoretical Physics of MIT for the kind hospitality. This work has been supported in part by Conselho Nacional de Desenvolvimento Científico e Tecnológico (CNPq), Brazil, by Fundação de Amparo à Pesquisa do Estado de São Paulo (FAPESP), Brazil, and by Coordenação de Aperfeiçoamento de Pessoal do Ensino Superior (CAPES), Brazil. The computer work was realized mostly in the workstations of the Department of Mathematical Physics, which were acquired through grants from FAPESP/CNPq.

(a) On leave of absence from Instituto de Física, Universidade Federal do Rio Grande do Sul, Caixa Postal 15051, 91500-Porto Alegre, RS, Brazil.

(b) On leave of absence from Instituto de Física, Universidade de São Paulo, Caixa Postal 20516, 01498 São Paulo, SP, Brazil.

[1] H. O. Girotti, M. Gomes, and A. J. da Silva, Phys. Lett. B 274, 357 (1992).
[2] S. Deser, R. Jackiw, and S. Templeton, Ann. Phys. (N.Y.) 140, 372 (1982).

[3] In Eq. (1), $e$ is the fermion charge, $\mathbf{r}$ is the relative distance between electrons, $r=|\overrightarrow{\mathrm{r}}|, L=\overrightarrow{\mathrm{r}} \times \overrightarrow{\mathrm{p}}$ is the orbital angular momentum, whose eigenvalues are denoted by $l$, and $\vec{p}$ is the relative linear momentum of the electrons. The fermion and topological masses are $m$ and $\theta$, respectively, while $K_{0}$ and $K_{1}$ designate the modified Bessel functions. The linear dependence of $V$ on $L$ accounts for the breaking of parity and time-reversal invariance in the nonrelativistic approximation.

[4] The potential (1) looks similar to the one derived by Ya. I. Kogan (Pis'ma Zh. Eksp. Teor. Fiz. 49, 194 (1989) [JETP Lett. 49, 225 (1989)]) for the same problem. There are some important differences, however, that we would like to stress. The derivation in Ref. [1] is based solely on relativistic quantum field theory. [For a similar derivation of the effective electron-electron potential arising from $\mathrm{QED}_{4}$ see, for instance, J. J. Sakurai, Advanced Quantum Mechanics (Addison-Wesley, Reading, MA, 1967); A. I. Akhiezer and V. B. Berestetskii, Quantum Electrodynamics (Wiley, New York, 1965).] On the other hand, in the work of Kogan the corresponding potential is determined in two steps. First, the $A_{\mu}$ vector created by a point charge is computed. Then, the quantum mechanical Hamiltonian describing the low-energy relative motion of the two electrons is assumed to be that of a charged particle in the presence of the $A_{\mu}$ external field. As a consequence, an extra term proportional to $\left[1-|\theta| r K_{1}(|\theta| r)\right]^{2}$ arises in Kogan's formulation.

[5] F. Bloch and A. Nordsieck, Phys. Rev. 52, 54 (1937).

[6] N. N. Bogoliubov and D. V. Shirkov, Introduction to the Theory of Quantized Fields (Wiley, New York, 1980).

[7] A. V. Svidzinskiy, Zh. Eksp. Teor. Fiz. 31, 324 (1956) [Sov. Phys. JETP 4, 179 (1957)].

[8] This does not constitute a serious limitation for $\mathrm{QED}_{4}$, as far as the infrared structure of the theory is concerned. In fact, in that case these diagrams are, on the one hand, free of infrared singularities while, on the other hand, they do not give rise to a mechanism for dynamical mass generation.

[9] A. N. Redlich, Phys. Rev. Lett. 52, 18 (1983); Phys. Rev. D 29, 2366 (1984).

[10] Throughout this paper we use natural units $(c=\hbar=1)$. Our metric is $g_{00}=-g_{11}=-g_{22}=1$, while for the $\gamma$ matrices we adopt the representation $\gamma^{0}=\sigma_{3}, \quad \gamma^{1}=i \sigma^{1}$, $\gamma^{2}=i \sigma^{2}$, where $\sigma^{i}, i=1,2,3$, are the Pauli spin matrices.

[11] For the vertex corrections in the MCS theory see I. Kogan, Phys. Lett. B 262, 83 (1991); I. Kogan and G. W. Semenoff, Nucl. Phys. B368, 718 (1992).

[12] Hence, only the minimum energy eigenvalue $(n=0)$, for a given $l$, can be found through this method. 